\title{
Chloroplast Research Methods: Probing The Targeting, Localization And Interactions Of Chloroplast Proteins
}

Paul Jarvis ${ }^{1}$

${ }^{1}$ Department of Plant Sciences, University of Oxford

\section{Corresponding Author}

Paul Jarvis

paul.jarvis@plants.ox.ac.uk

\section{Date Published}

August 15, 2019

\section{Editorial}

Chloroplasts are the organelles that define plants ${ }^{1}$. Along with many other metabolic, developmental and signaling functions, chloroplasts are responsible for photosynthesis - the process whereby sunlight energy is harnessed to power the cellular activities of life. Consequently, chloroplasts are essential, not only for plants but also for the myriad of ecosystems that depend on plants, and for agriculture. Chloroplasts are composed of thousands of different proteins, most of which are nucleus-encoded and imported from the cytosol before being routed internally to one of a number of clearly-distinct intraorganellar compartments ${ }^{1}$. To achieve a more complete understanding of chloroplast development and functions, and to enable biotechnological strategies involving chloroplast manipulation that address global challenges linked to food security or bioenergy, it will be essential to determine the targeting, localization and interactions of important chloroplast proteins. This method collection describes a set of critically important and complementary techniques that may be used to accomplish these goals. The collection mostly focuses on the widelyused model plant Arabidopsis thaliana (thale cress), but

\section{Citation}

Jarvis, P. Chloroplast Research Methods: Probing The Targeting, Localization And Interactions Of Chloroplast Proteins. J. Vis. Exp. (150), e59935, doi:10.3791/59935 (2019).
DOI

$10.3791 / 59935$
URL

jove.com/video/59935 the methods may also be adapted and applied to other organisms.

The collection includes descriptions of two different techniques for analyzing the import of nucleus-encoded proteins into chloroplasts across the double-membrane envelope. The article by Ling and Jarvis ${ }^{2}$ describes an in vitro method in which isolated chloroplasts are incubated with a radioactively-labelled precursor protein. The extent to which the chloroplasts take up the precursor protein is determined by monitoring the protein size change that occurs as a result of transit peptide (targeting leader sequence) cleavage, using SDS-PAGE and phosphor-imaging. The presented method is a development of an approach that has been used to study chloroplast protein import in vitro for several decades ${ }^{3,4}$, and incorporates additional steps that enable assessment of the responsiveness of the import machinery to stress conditions experienced by the plant ${ }^{5}$. On the other hand, the article by Lee et al. ${ }^{6}$ describes an in vivo method based on the transient expression of a chimeric precursor protein, carrying a fluorescent protein domain, in intact cells (protoplasts). In this assay, the extent of chloroplast protein import can be followed in two different ways: by monitoring the localization and intensity of the fluorescence 
signal using fluorescence microscopy; and, by analyzing the protein size change that occurs as a result of transit peptide cleavage using immunoblotting. These two methods are highly complementary, and can deliver compelling results when used together in parallel ${ }^{5}$.

Once a protein has been imported across the envelope into the chloroplast, and its transit peptide has been removed, it may either take on its final conformation in the stroma (the main internal aqueous compartment of the organelle), or engage one of a number of internal sorting pathways ${ }^{1}$. As the site of the highly-abundant photosynthetic complexes, the thylakoid membranes are a major destination for such internal sorting; in fact, thylakoid protein targeting involves multiple, mechanistically-distinct pathways. The article by Asher et al. ${ }^{7}$ describes a range of in vitro methods that enable different thylakoid protein translocation pathways to be studied. These methods involve the incubation of isolated thylakoids with radioactively-labelled precursor protein and, in some cases, a concentrated stromal extract. The extent to which the protein is taken up by the thylakoids is monitored by assessing cleavage of the targeting signal, and protection of the protein from exogenously-applied thermolysin protease, using SDSPAGE and phosphor-imaging. Of course, the thylakoids are not the only subcompartment of the chloroplast, and it is often desirable to have the ability to assess other compartments as well. In this regard, the article by Bouchnak et al. $^{8}$ is particularly important, as it describes methods for the subfractionation of chloroplasts to produce highlypure samples corresponding to the envelope membranes, the stroma, and the thylakoids. Once prepared, these fractions may be analyzed by immunoblotting and/or mass spectrometry, in order to provide a wealth of information on the suborganellar localization of chloroplast proteins ${ }^{9}$.
With regard to the analysis of protein-protein interactions and multiprotein complex assemblies, two different methodologies are described in the collection. The article by Shanmugabalaji et al. ${ }^{10}$ presents a method for the affinity purification of chloroplast multiprotein complexes. This technique involves the analysis of transgenic plants expressing a component of the complex of interest that has been engineered to carry an affinity tag (the socalled tandem affinity purification tag, or TAP tag). The ability of this tag to bind strongly to an otherwise inert matrix is exploited as part of the purification strategy. The presented method focuses specifically on purification of the chloroplast protein import machinery (this comprises multiprotein complexes called TOC and TIC embedded in the envelope membranes ${ }^{1}$ ), but in principle it can be adapted to study any of the other multiprotein assemblies that exist in chloroplasts $^{11}$. The article by Rantala et al. ${ }^{12}$ describes a complementary approach to complex characterization based on electrophoresis under native conditions. The technique, as presented here, involves the liberation of photosynthetic complexes from purified thylakoids using mild, non-ionic detergent, followed by their separation using blue native (BN)PAGE. Primary resolution of the complexes may be followed by a second dimension of electrophoresis under denaturing conditions, and this enables visualization of the individual components of each complex identified in the first dimension. As with the TAP method, this native PAGE approach can be successfully adapted to study other protein complexes within the organelle ${ }^{13,14}$. With either approach, the purified complexes may be analyzed in a variety of ways, including by immunoblotting and mass spectrometry.

Together, the articles included in this methods collection present a powerful set of complementary techniques that, in conjunction with existing resources ${ }^{15,16}$, may be employed 
to greatly improve our understanding of diverse aspects of chloroplast biogenesis and function, particularly those closely linked to the organellar proteome. Because chloroplasts are responsible for the bulk of terrestrial photosynthetic primary production, and furthermore have vital roles in the responses of plants to the environment (including both biotic and abiotic stresses), these remarkable organelles will inevitably remain a major focus of fundamental and applied research across the globe for years to come.

\section{Disclosures}

Commercial application of the author's research is covered by patent applications GB1803833.1, GB1803834.9, GB1815206.6 and US 16/643507.

\section{Acknowledgments}

Research in the author's laboratory is funded by the Biotechnology and Biological Sciences Research Council (BBSRC; grant references BB/N006372/1, BB/R005591/1, BB/R009333/1, BB/R016984/1).

\section{References}

1. Jarvis, P., López-Juez, E. Biogenesis and homeostasis of chloroplasts and other plastids. Nature Reviews Molecular Cell Biology. 14 (12), 787-802 (2013).

2. Ling, Q., Jarvis, P. Analysis of protein import into chloroplasts isolated from stressed plants. Journal of Visualized Experiments. (117) (2016).

3. Aronsson, H., Jarvis, P. A simple method for isolating import-competent Arabidopsis chloroplasts. FEBS Letters. 529 (2-3), 215-220 (2002).
4. Fitzpatrick, L. M., Keegstra, K. A method for isolating a high yield of Arabidopsis chloroplasts capable of efficient import of precursor proteins. Plant Journal. 27 (1), 59-65 (2001).

5. Ling, Q., Jarvis, P. Regulation of chloroplast protein import by the ubiquitin E3 ligase SP1 is important for stress tolerance in plants. Current Biology. 25 (19), 2527-2534 (2015).

6. Lee, J., Kang, H., Hwang, I. Studying protein import into chloroplasts using protoplasts. Journal of Visualized Experiments. (142) (2018).

7. Asher, A., Ganesan, I., Klasek, L., Theg, S. M. Isolation of physiologically active thylakoids and their use in energy-dependent protein transport assays. Journal of Visualized Experiments. (139) (2018).

8. Bouchnak, I., Moyet, L., Salvi, D., Kuntz, M., Rolland, N. Preparation of chloroplast sub-compartments from Arabidopsis for the analysis of protein Localization by immunoblotting or proteomics. Journal of Visualized Experiments. (140) (2018).

9. Salvi, D. et al. AT_CHLORO: the first step when looking for information about subplastidial localization of proteins. Methods in Molecular Biology. 1829 395-406 (2018).

10. Shanmugabalaji, V., Douet, V., Agne, B., Kessler, F. Affinity purification of chloroplast translocon protein complexes using the TAP tag. Journal of Visualized Experiments. (141) (2018).

11. Kikuchi, S. et al. A Ycf2-FtsHi heteromeric AAA-ATPase complex is required for chloroplast protein import. Plant Cell. 30 (11), 2677-2703 (2018). 
12. Rantala, M., Paakkarinen, V., Aro, E. M. Analysis of thylakoid membrane protein complexes by blue native gel electrophoresis. Journal of Visualized Experiments. (139) (2018).

13. Kikuchi, S., Hirohashi, T., Nakai, M. Characterization of the preprotein translocon at the outer envelope membrane of chloroplasts by blue native PAGE. Plant and Cell Physiology. 47 (3), 363-371 (2006).

14. Chen, L. J., Li, H. M. Stable megadalton TOC-TIC supercomplexes as major mediators of protein import into chloroplasts. Plant Journal. 92 (2), 178-188 (2017).

15. Jarvis, R. P. in Methods in Molecular Biology. Vol. 775 (ed John M. Walker) 432, Humana Press, New York City, (2011).

16. Jarvis, R. P. in Methods in Molecular Biology. Vol. 774 (ed John M. Walker) 374, Humana Press, New York City, (2011). 